\title{
Tim-4 in Health and Disease: Friend or Foe?
}

\author{
Wen Liu ${ }^{1 t}$, Liyun $\mathrm{Xu}^{1,2+}$, Xiaohong Liang ${ }^{1}$, Xiaojun Liu ${ }^{3}$, Yangbing Zhao ${ }^{3,4}$, Chunhong Ma ${ }^{1 *}$ \\ and Lifen $\mathrm{Gao}{ }^{\text {* }}$
}

${ }^{1}$ Key Laboratory for Experimental Teratology of Ministry of Education, Shandong Provincial Key Laboratory of Infection and Immunology, Department of Immunology, School of Basic Medical Sciences, Shandong University, Jinan, China, ${ }^{2}$ Cell and Molecular Biology Laboratory, Zhoushan Hospital, Zhoushan, China, ${ }^{3}$ Center for Cellular Immunotherapies, University of Pennsylvania Cancer Center, Philadelphia, PA, United States, ${ }^{4}$ Department of Pathology and Laboratory Medicine, Perelman School of Medicine, University of Pennsylvania, Philadelphia, PA, United States

\section{OPEN ACCESS}

Edited by:

Catherine Sautes-Fridman, INSERM U1138 Centre de Recherche des Cordeliers, France

Reviewed by:

Masaki Terabe,

National Institutes of Health (NIH), United States

Heung Kyu Lee,

Korea Advanced Institute of Science and Technology, South Korea

Rosemarie DeKruyff,

Stanford University, United States

${ }^{*}$ Correspondence:

Chunhong Ma

machunhong@sdu.edu.cn

Lifen Gao

glfflg@sdu.edu.cn

tThese authors have contributed equally to this work

Specialty section:

This article was submitted to

Cancer Immunity and Immunotherapy,

a section of the journal

Frontiers in Immunology

Received: 19 November 2019 Accepted: 09 March 2020

Published: 02 April 2020

Citation:

Liu W, Xu L, Liang X, Liu X, Zhao Y, Ma $C$ and Gao L (2020) Tim-4 in Health and Disease: Friend or Foe?

Front. Immunol. 11:537.

doi: 10.3389/fimmu.2020.00537
T-cell immunoglobulin and mucin domain containing 4 (Tim-4) is a phosphatidylserine receptor and is selectively expressed on antigen presenting cells. Recently, Tim-4 was reported to be expressed on iNKT cells, B1 cells, and tumor cells, suggesting it has multiple biological functions. In this review, we mainly summarize the expression and regulation of Tim-4 in immune cells including $T$ cells, macrophages, dendritic cells, NKT cells, B cells, and mast cells. The expression of Tim-4 in these cells implies that Tim-4 might participate in immune related diseases. Emerging evidence emphasizes a substantial role for Tim-4 in maintaining homeostasis by regulating various immune responses, including viral infection, allergy, autoimmunity, and tumor immunity. Here, we collectively evaluated the role of Tim-4 in health and diseases. This summary will be extremely useful to fully understand the function of Tim-4 in the pathogenesis of immune related diseases, which would provide novel clues for the diagnosis and treatment of diseases.

Keywords: Tim-4, macrophages, $\mathrm{T}$ cells, phosphatidylserine, immune regulation

\section{INTRODUCTION}

The $\mathrm{T}$ cell immunoglobulin domain and mucin domain (Tim) gene family is a relatively new gene family and was discovered in 2001 (1). Totally, the Tim family contains three members (TIM-1, TIM-3, TIM-4) in humans, and eight members (Tim-1 to Tim-8) in mice. The Tim gene family is an attractive area of research due to its location on the mouse chromosome 11B1.1 or human chromosome 5q33.2, which is related to asthma, allergic diseases, and autoimmune diseases (2-4). Unlike other Tim molecules, Tim-4 is mainly expressed on antigen-presenting cells (APCs) but not on T cells (5). In addition, Tim-4, identified as a natural ligand of Tim-1, can modulate T cell proliferation, which is involved in the development of multiple immune diseases $(6,7)$.

Shortly after the discovery of the Tim gene family, in 2004, Shakhov et al. found a selectively downregulated gene named SMUCKLER (Spleen, Mucin-Containing Knockout of Lymphotoxin) in $L T-\alpha$ and $L T-\beta$ deficient mice (8). This gene encodes a $60 \mathrm{kDa}$ type I transmembrane protein including a signal peptide, a characteristic of immunoglobulin variable region-like (IgV) domain, a mucin-like region, a transmembrane region, and an intracellular tail region, which was later identified as Tim-4. The IgV-like domain contains a conserved Arginine-Glycine-Aspartic acid (RGD) motif, which can bind to integrin proteins. The mucin-like domain of Tim-4 is the longest of the Tim family members and is rich in threonine, serine, and proline 
residues. The intracellular region of Tim- 4 contains about $42-$ 77 amino acids, which is the most highly conserved region of mouse and human homologs. In particular, the intracellular domain of Tim-4 lacks tyrosine activation residues. Therefore, the expression profile and special structure of Tim-4 might indicate its intriguing role in health and diseases.

\section{INTRODUCTION TO TIM-4}

Tim-4 is highly expressed in peripheral lymphoid tissues, such as the tonsils, thymus, spleen, lymph nodes (LNs), and Peyer's nodule but is poorly expressed in the lung, liver, and kidney tissues $(5,9,10)$. It has been confirmed that Tim- 4 is selectively expressed in macrophages, mature dendritic cells (DCs), peritoneal B1 cells, and invariant natural killer T (iNKT) cells (9, 11). Furthermore, the levels of Tim-4 expression can be regulated by different stimulators, such as lipopolysaccharide (LPS), cholera toxins, cytokines, Concanavalin A (ConA), and danger associated molecular patterns (DAMPs) (12-16). However, Tim4 expression can also be downregulated under some conditions. It is reported that probiotics such as Bifidobacterium infantis reduced Tim- 4 expression in DCs by inhibiting its transcription factor, STAT6 (17). Vitamin D (VitD) might repress the Tim4 gene transcription and expression via VitD receptor (18). In addition to expression in immune cells, Tim-4 was also found to be ectopically expressed in tumor cells, including lung cancer (19), colorectal cancer (20), juvenile xanthogranuloma, tissue sarcomas, Langerhans cell sarcoma, and parapharyngeal liposarcoma (21).

Specific microenvironment modulates Tim-4 expression. We observed that Tim-4 expression was relatively low in lung cancer cell lines, while its expression was increased in lung cancer tissues. Furthermore, Tim-4 expression was greatly enhanced by IL-6 or TGF- $\beta$, which were highly abundant in tumor microenvironment $(19,22,23)$. Our studies also showed that microenvironment of non-alcohol fatty liver disease increased Tim-4 expression in liver tissues greatly, especially in macrophages (24). These data suggest that Tim-4 is inducible under specific disease conditions. However, the mechanisms governing Tim-4 expression remain largely elusive. Some evidences suggested that the histone acetyltransferase, p300, and STAT6 regulate the expression of Tim-4 in DCs upon stimulation by the cholera toxin (25). Recently, it was found that cigarette smoke extract (CSE) upregulated Tim-4 expression in immature DCs from murine bone marrow, and the upregulation of Tim-4 stimulated by CSE was inhibited by an ERK inhibitor but not by a p38 or JNK inhibitor (26). Further research is required to elucidate the mechanisms driving transcriptional and translational regulation of Tim- 4 .

Emerging evidences showed that Tim-4-Ig could bind to T cells, suggesting that Tim-4 receptors might exist on the surface of $\mathrm{T}$ cells. It was subsequently shown that Tim-4-Ig bound with CHO cells transfected with Tim-1, but not Tim-3 or Tim-4, confirming the interaction between Tim-4 and Tim-1 (5). In addition, Tim-4-Ig bound with activated T cells, which expressed high levels of Tim-1, and this binding was blocked by antibodies against Tim-1, suggesting that Tim-4 is indeed a natural ligand of Tim-1 (5). Another study showed that Tim-4-Ig showed high affinity for early and late apoptotic Jurkat cells, but not living cells (10). Subsequent results demonstrated that Tim-4 could bind to phosphatidylserine (PS) exposed on the surface of apoptotic cells. Therefore, Tim- 4 was identified as another receptor of PS (27). Besides, they found that both Tim-1 and Tim- 4 bound PS exposed on apoptotic cells or exosomes, which led to the realization that the Tim-1-Tim-4 interaction occurred through a PS bridge. Thus, the interaction between Tim- 1 and Tim- 4 is indirect (27). In addition, leukocyte mono-immunoglobulin (Ig)like receptor 5 (LMIR5) also interacted with Tim-4, suggesting that Tim-4 is a possible ligand for LMIR5. Nevertheless, LMIR5 neither bound to PS nor affected Tim-4-mediated phagocytosis of apoptotic cells (28). Of course, there might be other unidentified receptors of Tim- 4 .

\section{REGULATION OF TIM-4 ON IMMUNE CELLS}

\section{T Lymphocytes}

It is reported that Tim- 4 mRNA is not expressed in $\mathrm{T}$ cells, however, Tim-4 displays modulation on $\mathrm{T}$ cells through its receptor. Rodriguez-Manzanet et al. found that the proliferation of $\mathrm{T}$ cells incubated with Tim-4-expressing $\mathrm{CHO}$ cells was significantly higher than that of control cells (29). Blockade with a Tim-4 antibody partially inhibited $\mathrm{T}$ cell proliferation induced by Tim-4-expressing CHO cells, suggesting that Tim-4 is involved in promoting $\mathrm{T}$ cell activation. $\mathrm{CHO}$ cells also express endogenous co-stimulatory molecules, and these molecules, in conjunction with Tim-4, may play a synergistic role in T cell activation (29). Interestingly, the regulation of Tim- 4 on $\mathrm{T}$ cells has two sides. The dose of Tim- 4 is critical for its effect on T cells. Higher doses of Tim-4-Ig promoted $\mathrm{T}$ cell proliferation and amplification in vitro and in vivo, while lower concentrations of Tim-4-Ig strongly inhibited $\mathrm{T}$ cell proliferation induced by anti-CD3 and antiCD28 antibodies (5). Moreover, Tim-4 exerts bimodal functions depending on the activation status of $\mathrm{T}$ cells. It was reported that Tim- 4 bound to naïve $\mathrm{CD}_{4}^{+} \mathrm{T}$ cells that did not express Tim1 , and Tim-4 inhibited naïve $\mathrm{CD} 4^{+} \mathrm{T}$ cell proliferation but not of pre-activated T cells (7). However, Cao et al. found that Tim4 Fc could inhibit both naïve and pre-activated T-cell activation, proliferation and cytokine production via a Tim-1-independent pathway (30). These inconsistent findings might attribute to different experimental conditions. In addition, Ge et al. reported that Tim-4 could also bind Tim-3 on the surface of polarized T helper type 1 (Th1) cells to increase p300 phosphorylation in Th1 cells, which further increased the levels of Fas ligand in the cells and induced Th1 cell apoptosis (31). These data suggest that Tim- 4 might regulate $\mathrm{T}$ cell activation by cross-linking different receptor, and unknown receptors on $\mathrm{T}$ cells are required to be identified.

It is well-known that the MAPK pathway is essential for T cell proliferation and differentiation (32-34). Tim-4 Ig could induce Akt and ERK phosphorylation of $\mathrm{T}$ cells by interacting with Tim-1. Tim-4-Ig induced Akt phosphorylation and increased 
Bcl-2 protein expression were involved in the protection of activated $\mathrm{T}$ cells from apoptotic cell death. However, the presence of Tim-4-Ig did not increase Bcl-xL protein expression (29). Thus, it is possible that Tim- 4 promotes $\mathrm{T}$ cell proliferation by induction of cell division and anti-apoptotic signals. However, Tim-4-Fc inhibited ERK phosphorylation of T cells stimulated with coated anti-CD3 and anti-CD28 (7), suggesting that Tim4-Fc could also inhibit the MAPK pathway even in pre-activated T cells expressing Tim-1. Cao et al. also demonstrated that Tim4 Fc could inhibit the MAP kinase pathway in T cells. Tim-4 Fc not only inhibited $\mathrm{T}$ cell activation but also prevented the differentiation of Th17 cells, even blocked IL-17 production in Th17-polarized cultures (30). This study also showed that both $\mathrm{IgV}$ and mucin domains were required for Tim- 4 mediated inhibition of T-cell activation and Th17 differentiation via the MAPK pathway (30).

\section{Macrophages}

We reported that Tim-4 inhibited the expression of CD80, CD86, MHC-II and the production of TNF- $\alpha$ in LPS-treated macrophages (15). Tim-4 could also suppress nitric oxide (NO) production in LPS- or IFN- $\gamma$ - activated macrophages (14). Furthermore, Tim-4 was shown to protect mice from ConA-induced liver injury or LPS-induced septic shock by inhibiting pro-inflammatory cytokines such as IL-6 and TNF$\alpha(15,35)$. Although Con A-induced liver injury is a T cellmediated autoimmune hepatitis model (36), it has been found that Kupffer cells are involved in the initiation and propagation of liver damages (37), suggesting that Tim-4 expressed on macrophages might attenuate the priming and activation of $\mathrm{T}$ cell responses in Con A-induced hepatitis. Further analysis showed that Tim-4 inhibited cytokines and NO production via the NF$\kappa \mathrm{B}$ or Jak2/STAT1 signaling pathways (14). Taken together, we speculate that Tim-4 may play an inhibitory role in the course of macrophage activation.

Another important function of macrophages is phagocytosis, which plays an essential role in the elimination of apoptotic cells to maintain homeostasis. It is known that PS exposure on the cell surface is the "eat me" signal of apoptotic cells for engulfment and removal called efferocytosis (38). Tim-4 enhances Tyro3, Axl, and Mer receptors (TAMs)-stimulated efferocytosis in resident peritoneal macrophages, Kupffer cells, and $\mathrm{CD} 169^{+}$skin macrophages, but not in thioglycollate-elicited peritoneal macrophages or primary cultured microglia, which do not express Tim-4 (39). Both Kobayashi et al. and Miyanishi et al. identified Tim-4 as a PS receptor for the engulfment of apoptotic cells, and the phagocytosis of apoptotic thymocytes was decreased when peritoneal macrophages were pretreated with Tim-4-blocking antibody $(10,27)$. Santiago et al. found that crystal structures of Tim-4 had a metal-ion-dependent ligand binding site (MILIBS) for PS binding, and any amino acid deficiency or mutation in the MILIBS region of Tim-4 would lead to impaired phagocytosis (40). Tietjen et al. further clarified that the sensitivity of Tim-4 to recognize PS was related to the surface density of PS, as well as the composition and the fluidity of the membrane (41).
Park et al. explored the downstream pathway for the recognition of PS by Tim-4. They declared that Tim-4 was independent of the two known engulfment signaling pathways: the ELMO1/Dock180/Rac pathway and GULPmediated pathway (42). In contrast, it was found that the transmembrane region of Tim-4 was dispensable for Tim-4mediated engulfment, suggesting that the potential membrane molecule binding to the extracellular region of Tim-4 could coordinate the signaling together with Tim-4. As expected above, Flannagan et al. found that integrin proteins bound to Tim- 4 as a co-receptor to induce the signal transduction cascade required for phagocytosis of apoptotic cells (43). Recently, Lee et al. identified Fibronectin (Fn1) as a novel Tim-4-associating protein, and found that Fn1 acted as a scaffold to form the complex composed of Tim-4 and integrin to mediate efferocytosis (44).

Interestingly, Tim-4 emerges as a key marker of resident macrophage in various tissues. Recruited macrophages do not express Tim-4, highlighting that Tim-4 is required for a specific population of macrophages. At steady state, Tim- $4^{+}$ resident cardiac macrophages, which were $\mathrm{LYVE}^{+} \mathrm{MHC}$ $\mathrm{II}^{\text {low }} \mathrm{CCR} 2^{-}$, maintained self-renewal despite low input of blood monocytes. Following myocardial infarction, macrophages promoted both injury and repair, while resident cardiac macrophages played a non-redundant, cardioprotective role by limiting adverse remodeling (45). In the intestine, tissue-resident macrophages were long lived and defined by Tim-4 and CD4 expression $(46,47)$. In lean, murine visceral adipose tissues, the majority of adipose tissueresident $\mathrm{F} 4 / 80^{\text {hi }}$ macrophages (ATM) expressed Tim-4, and embryonic-derived $\mathrm{Tim}-4^{+} \mathrm{MHCII}^{\text {low }}$ and $\mathrm{Tim}-4^{+} \mathrm{MHCII}^{+}$ ATM subsets were long-lived (48). Likewise, Tim-4 was essential for the maintenance of resident peritoneal macrophage homeostasis (9).

\section{Dendritic Cells}

DCs are the most potent antigen-presenting cells to initiate a naïve T cell response. Yeung et al. (49) reported that the blockade of Tim- 4 on the surface of DCs could promote the transformation of naïve $\mathrm{CD}^{+} \mathrm{T}$ cells to induced regulatory $\mathrm{T}$ cells (iTreg) in skin allografts both in vitro and in vivo. Li et al. (50) found that DC infiltration and Tim-4 expression were increased in hepatic warm ischemia reperfusion (IR) models, and Tim-4 blockade on DCs significantly attenuated hepatic injury and reduced the release of pro-inflammatory cytokines. They found that Tim-4 blockade inhibited Th2 cell differentiation and facilitated induced $\mathrm{CD}^{+}{ }^{+} \mathrm{CD} 25^{+}$Foxp $^{+}$iTreg generation through the IL-4/STAT6 signaling pathway in vitro. In vivo data showed that adoptive transfer of iTreg induced by Tim- 4 blockade into IR mouse models remarkably attenuated liver injury. Therefore, Tim-4 on DCs played a critical role in mediating hepatic IR injury. In a model of allergy-induced colitis, it was found that Tim-4 expression on DCs was increased greatly (51). However, its role in colitis remains unknown. Collectively, these studies identify a key role for Tim-4 in immune response by regulating DCs, suggesting that Tim-4 might be an efficient target for the prevention of IR injury in the liver or other tissues. 
Recently, Zhang et al. (52) found that the expression of Tim-4 was variable in the different subsets of DCs in skin and skindraining LNs that were studied. Dermal CD207 ${ }^{+}$DCs and LNs resident $\mathrm{CD}_{207}{ }^{-} \mathrm{CD} 4^{+}$DCs highly expressed Tim-4. In Tim4-deficient mice, it was found that loss of Tim-4 significantly upregulated the population of epidermal Langerhans cells and LNs resident CD $207^{-} \mathrm{CD} 4^{+}$DCs, which was consistent with that in macrophages (53).

\section{Other Immune Cells}

Previously, it was shown that Tim-4 was expressed on thymic iNKT cells, and its expression was increased upon iNKT cell migration to the LNs. However, Tim-4 was dispensable for the development and function of iNKT cell at steady state (11). Tim-4 identified proinflammatory B effector 1 (Be1) cells, and Tim $-4^{+}$Be1 cells could decrease B16-F10 growth and metastasis in an IFN- $\gamma$-dependent manner (54). Moreover, Tim- $4^{+}$Be1 cells could promote pro-inflammatory Th cell differentiation in vivo and increase IFN- $\gamma$ production while decreasing IL-4, IL10, and Foxp3 expression. Importantly, blockade of Tim-4 could promote allograft tolerance, which was dependent on Be1 cell expression of Tim-4 (54).

Tim-4 is involved in mast cell activation. Tim- 4 crosslinking of Tim-1 enhanced cytokine production without affecting degranulation via signaling pathways downstream of FceRI in mast cells (55). In addition, stimulation with Tim-4 Ig also induced LMIR5-mediated activation of mast cells (28). Though mast cells did not constitutively express Tim-4, flagellin could induce mast cells to express Tim-4 by increasing STAT6 phosphorylation (56).

\section{THE ROLE OF TIM-4 IN DISEASES}

Since Tim-4 is closely related with immune regulation, it is likely that Tim- 4 is involved in numerous diseases by affecting the immune system.

\section{Allergy}

It is thought that misregulation of Th1/Th2 cells plays a critical role in the pathophysiology of asthma. Studies showed that several single nucleotide polymorphisms (SNPs) of Tim-1 were associated with asthma susceptibility in some populations (57, 58). In consideration of the associations between Tim-1 and Tim4, McIntire et al. presented the hypothesis that several SNPs in Tim-4 promoter might be associated with the susceptibility of asthma (1). Cai et al. reported an important relationship between the Tim-4-1419 G>A SNP and childhood asthma susceptibility in the Han population of China (59).

Cockroach allergen rBla $\mathrm{g} 7$, the crucial factor in cockroach allergy, enhanced Tim- 4 expression in DCs in a dose-dependent manner, suggesting that rBla g 7 challenged DCs induce Th2 polarization via Tim-4-, CD80-, and CD86-dependent mechanisms (60). Accumulating evidences showed that bacteriaassociated factors were also linked to the development of allergies (61). Consistently, the simultaneous exposure to cholera toxin and peanuts resulted in an increase in Tim-4, MHC II, and costimulatory molecules expression in DCs, which could induce differentiation and activation of peanut-specific Th2 cells in the intestine. However, blocking the interaction between Tim-4 and Tim-1 with antibodies inhibited the allergic reaction, indicating that modulating Tim-4 function may prove to be an efficient treatment for DC-induced peanut allergy in the future (12). In allergic rhinitis patients, VitD deficiency may contribute to the pathogenesis by increasing the Tim- 4 expression on DCs (18).

\section{Autoimmune Disease}

Autoimmune disease is characterized by the impaired structure and function of multiple tissues. Many factors contribute to the development of autoimmunity, including the deficient removal of apoptotic cells (62). Consequences of inefficient clearance of apoptotic bodies include the onset of autoimmune diseases, such as rheumatoid arthritis (RA) and systemic lupus erythematosus (SLE) (63). Tim-4 mediates the clearance of apoptotic bodies by macrophages, and plays a pivotal role in autoimmune diseases (64).

We reported that Tim-4 mRNA in PBMCs of SLE patients was significantly increased when compared to healthy controls. Additionally, Tim-4 was positively correlated with SLE (65). Though the correlation between two Tim-4 SNPs rs6874202, rs62382402 and SLE susceptibility was not found in a Chinese Han population, the GG genotype of the Tim- 4 gene at-1419 site might be associated with the disease activity of SLE (66). Miyanishi et al. demonstrated that mice lacking Tim-4 or MFGE8 (Milk Fat Globule EGF Factor VIII) developed little or no autoimmunity. In contrast, mice lacking both Tim-4 and MFG-E8 produced a high level of autoantibodies. Moreover, this process was accelerated by the administration of an anti-TNF- $\alpha$ antibody or pristane, a reagent that provokes type I IFN production (67). However, Rodriguez-Manzanet et al. found a significant development of auto-antibodies in Tim-4-deficiency mice on the B6 background (68). Although all mice were kept under sterile conditions, environmental factors might contribute to discrepancies in the experimental outcomes (69).

The associations between Tim-4 SNP rs7700944 and RA susceptibility were described in the Chinese Han and Hui populations, Zahedan, southeast Iran, and Egyptian population (70-72). However, in collagen-induced arthritis (CIA), Tim4 displayed dual function in the induction and effector phases. In the induction phase, anti-Tim- $4 \mathrm{mAb}$ exacerbated the development of CIA, while the arthritis scores and proinflammatory cytokines were reduced in anti-Tim- 4 treated mice at effector phase (6). In the induction of experimental autoimmune encephalomyelitis (EAE) model, anti-Tim-4 treatment greatly ameliorated the clinical feature of EAE (7). Thus, the role of Tim- 4 in autoimmunity is complicated, and reasonable therapy plans are being developed by targeting Tim-4.

\section{Tumor}

Baghdadi et al. found that Tim-4 was highly expressed on tumor related macrophages and DCs stimulated by DAMPs such as HMGB1, heat shock protein 90, monosodium urate, S100A8, or ATP (apyrase) secreted from dying or stressed tumor cells induced by chemotherapy. Upon ingestion of apoptotic B16F10 tumor cells triggered by chemotherapy, Tim-4-AMPK $\alpha 1$ 
interaction activated autophagy in macrophages, which could mediate degradation of ingested tumor cells, resulting in reduced antigen presentation and damaged cytotoxicity $\mathrm{T}$ lymphocyte responses (16). Xu et al. reported that glioma-derived macrophages, expressing high levels of Tim-4, contributed to tumor tolerance by phagocytizing T cells following PS exposure (73). Data from Tim-4 transgenic mice indeed demonstrated that overexpression of Tim- 4 on APCs resulted in decreased secondary $\mathrm{T}$ cell responses and decreased Ag-specific $\mathrm{T}$ cell numbers (74), thus Tim-4 played a negative role in antitumor immunity by inducing tumor immune tolerance. Consistently, a unique subset of $\mathrm{CD} 163^{+}$Tim $-4^{+}$resident omental macrophages was defined to be responsible for metastatic spread of ovarian cancer cells recently. Moreover, $\mathrm{CD} 163^{+}$Tim $-4^{+}$tissue-resident macrophages promoted the circulating stem cell-like phenotype of ovarian cancer cells (75). Thus, the therapeutic manipulation of Tim-4 may provide a novel strategy to enhance antitumor immunity and boost cancer chemotherapy.

Experimental models of tumor revealed that combined treatment with anti-Tim-3 and anti-Tim-4 mAbs increased the efficacy of cancer vaccines (76). In accordance, Jinushi et al. revealed that a secreted PS-binding protein MFG-E8 blockade also favored the establishment of an immunogenic tumor microenvironment (77). Consistent with this issue, novel monoclonal antibodies targeting Tim-4 could enhance the curative effects of vaccinations against melanomas in B16 mice. Blocking Tim-4 increased vaccine-induced antitumor responses against irradiated B16 melanoma mice by increasing the numbers of $\mathrm{CD}^{+} \mathrm{T}$ cells and effector functions (78). Interestingly, a recent study indicated that an siRNA targeting the FG-CC' loop could enhance the therapeutic effects of DC vaccines against gastric cancer, suggesting that targeting the FG-CC' loop in Tim-4 might be helpful in the development of novel immunotherapeutic treatments (79).

Recently, ectopic expression of Tim-4 in tumor tissues was discovered, and Tim- 4 was shown to act as an oncogene. We found that Tim-4 expression was significantly higher in nonsmall-cell lung cancer (NSCLC) tissues, and overexpression of Tim-4 was associated with adverse prognosis. Mechanistically, overexpression of Tim-4 promoted lung cancer cell growth and proliferation, depending on its Arg-Gly-Asp (RGD) motif of IgV domain. Furthermore, Tim- 4 could interact with $\alpha v \beta 3$ integrin through RGD motif (19). In addition, we found that Tim-4 also promoted migration, invasion, and epithelial mesenchymal transformation of NSCLC, which was responsible for IL-6 mediated metastasis of lung cancer (80). Li et al. found that Tim-4 expression was also significantly increased in glioma tissues. In the human glioma cell line LN-18 cells, Tim- 4 was shown to promote growth, suppress apoptosis, and enhance clonogenicity (81). Tim-4 was upregulated in colorectal cancer and promoted the growth of colorectal cancer by activating angiogenesis and recruiting tumor-associated macrophages via the PI3K/AKT/mTOR signaling pathways (20).

The above data indicate that targeting Tim-4 might simultaneously reverse immune tolerance and suppress tumor cell growth and migration, which would provide a promising tool for tumor therapy. Therefore, it is required to specifically evaluate immune regulatory drugs such as Tim-4 inhibitors to define their anti-tumorigenic actions during tumorigenesis $(82,83)$. However, no effective Tim-4 inhibitors have been developed to date.

\section{Pathogen Infection}

More recent studies revealed that Tim family members played critical roles in enveloped viral infections possibly by interacting with virion-associated PS. It was found that various enveloped viruses could expose PS on their membranes. Tim-1 and multiple enveloped viruses were dependent on PS as demonstrated by blocking liposome and Tim-1 mutagenesis experiments, and the function of additional PS receptors especially hTim4 paralleled with hTim-1 (10). Similar to Tim-1, residues in the PS binding pocket of murine and human Tim-4 were found to be important for Ebola virus (EBOV) entry, and additional Tim-4-specific residues were also found to impact virion binding and internalization, which provided a greater understanding of the interaction between Tim-4 and EBOV virions (84). Interestingly, Tim-EBOV interactions were mechanically comparable to adhesion molecule-ligand interactions and the Tim-4-PS interaction was more resistant to mechanical force than the Tim-1-PS interaction (85). Exosomes from multiple sources increased HIV-1 entry into $\mathrm{T}$ cells and macrophages, and viral entry was potently blocked with anti-Tim-4 antibodies (86). In conclusion, hTim-4 and hTim1 significantly contributed to infection by diverse families of enveloped viruses, thereby making them an attractive therapeutic target for the intervention of viral infections in the future (87).

In addition, Tim- 4 contributed to efficient cell-to-cell spread by Listeria monocytogene $(\mathrm{Lm})$ in macrophages in vitro and growth of these bacteria was impaired in Tim- $4^{-/}$mice. Thus, Lm promoted its dissemination in a host by exploiting efferocytosis. This study suggests that PS-targeted therapeutics may be useful in the fight against infections by Lm and other bacteria that utilize similar strategies of cell-to-cell spread during infection (88). Also, we believe that antagonists of Tim-4 would be useful to block the spread of bacterial or viral infection.

\section{Chronic Metabolic Disease}

SNP site is the main content of genetic polymorphism study. Recently, genome-wide association studies or meta-analysis from different laboratories showed that some SNPs of Tim-4 gene were closely related to lipid metabolism. It was reported that TIMD4 SNP (rs1501908) was closely related to low density lipoprotein cholesterol (LDL-C) in populations from Korea and Europe (89, 90). It was also shown to be negatively correlated with low density lipoprotein (LDL) and total cholesterol (TC) and positively correlated with high density lipoprotein in a Chinese population (91). SNPs in the promoter region of TIMD4 rs6882076 was related to LDL, triglyceride and TC (92), and rs1553318 was also related to hyperlipidemia (93).

The level of Tim-4 mRNA expression in peripheral blood of patients with Type 2 diabetes mellitus was significantly increased and negatively correlated with LDL (94). The latest report found that blocking Tim- 4 could aggravate atherosclerosis in $l d l r^{-/-}$ mice (95), and the expression of Tim-4 mRNA was increased 


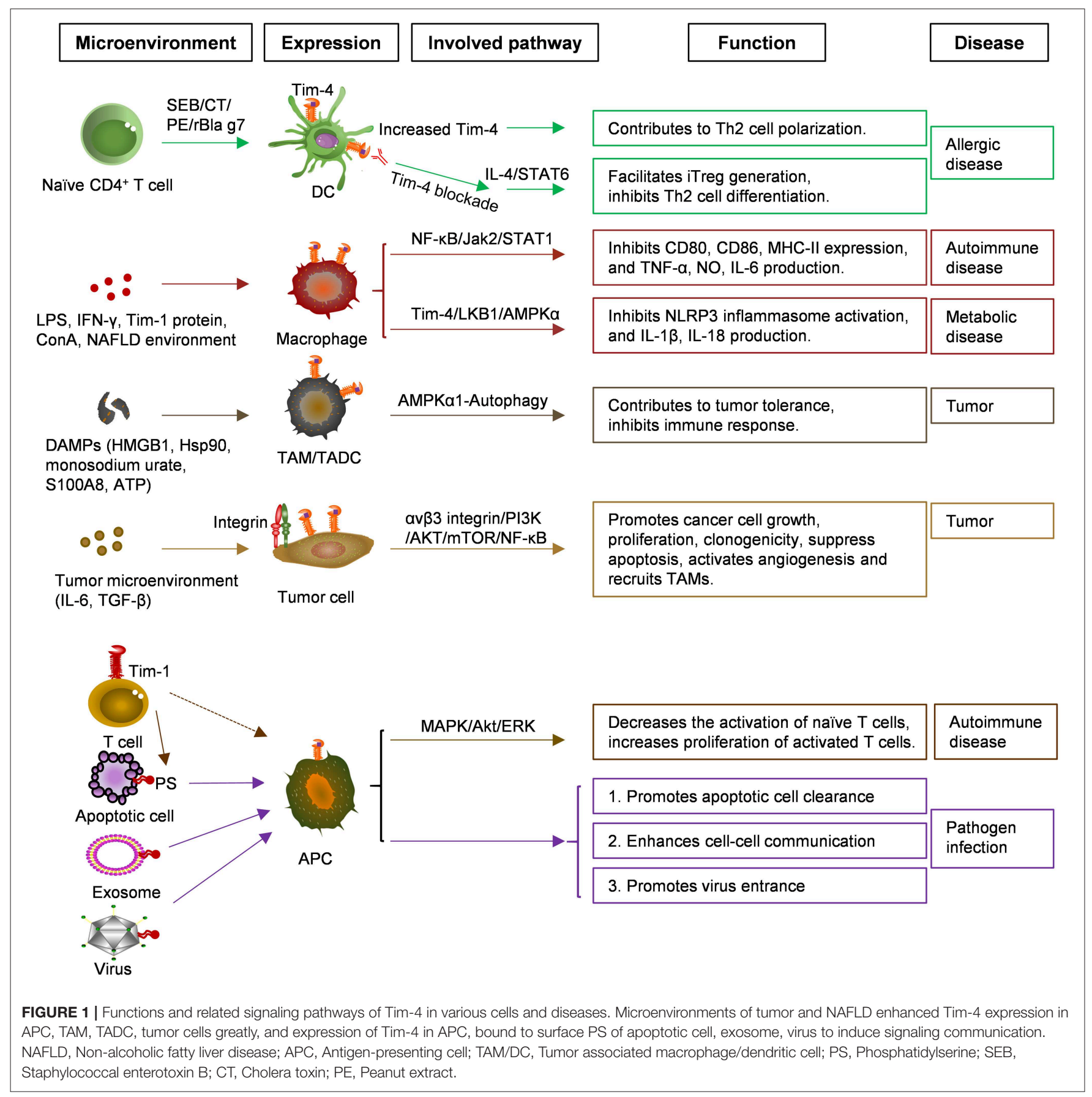

in liver tissues of rats on a high fat diet (96). Recently, we found that Tim- 4 knockout aggravated methionine and cholinedeficient diet induced NAFLD, suggesting the protective role of Tim- 4 in fatty liver disease. Furthermore, we revealed that Tim- 4 promoted AMPK $\alpha$ phosphorylation depending on LKB1. Tim4/LKB1/AMPK $\alpha$ interaction triggered autophagy and inhibited NLRP3 inflammasome activation, which might be responsible for NAFLD progress (24). However, we could not exclude whether Tim-4 was involved in lipid metabolism, a possibility that warrants further investigation.

\section{SOLUBLE TIM-4}

Tim-4 protein can be sheared into soluble Tim-4 (sTim-4) molecule by A Disintegrin and Metalloprotease 10 (ADAM 10) and ADAM 17, without affecting their binding to PS (97). sTim4 could be detected in serum or bodily fluids, and the changes of sTim-4 might indicate pathological conditions. Following a stroke, we found that sTim- 4 was significantly increased by days 2 and 5 . Furthermore, stroke severity was positively correlated with sTim-4 levels in plasma. Up-regulated sTim-4 in plasma might 
act as a prognostic biomarker of ischemic stroke (98). In addition, serum levels of sTim-4 were significantly increased in ankylosing spondylitis patients, which was positively correlated with TNF$\alpha$ levels and Bath ankylosing spondylitis disease activity index (99). However, we did not confirm the role of sTim-4 in vivo. We speculate that sTim- 4 might act as an antagonist of membrane surface Tim-4, which requires further investigation.

\section{DISCUSSION}

It was initially thought that Tim-4 was expressed only on APCs. However, in recent years, emerging evidence shows that Tim4 can also be expressed in other cells, such as iNKT cells, even in tumor cells, which provided a broad scope for Tim-4 research. Under stresses or DAMPs, Tim-4 is potentially induced in epithelial cells or other cell types to promote pathology. The analysis of the Tim- 4 crystal structure and the identification of its ligands provides a new pathway studying the role of Tim-4 in human diseases. Many findings show that the phagocytosis of apoptotic cells mediated by Tim- 4 might play an important role in autoimmune diseases. Therefore, Tim- 4 shows versatility in health and diseases (Figure 1). However, the exact role of

\section{REFERENCES}

1. McIntire JJ, Umetsu SE, Akbari O, Potter M, Kuchroo VK, Barsh GS, et al. Identification of Tapr (an airway hyperreactivity regulatory locus) and the linked Tim gene family. Nat Immunol. (2001) 2:1109-16. doi: 10.1038/ni739

2. Encinas JA, Kuchroo VK. Mapping and identification of autoimmunity genes. Curr Opin Immunol. (2000) 12:691-7. doi: 10.1016/S0952-7915(00)00164-3

3. Kuchroo VK, Umetsu DT, DeKruyff RH, Freeman GJ. The TIM gene family: emerging roles in immunity and disease. Nat Rev Immunol. (2003) 3:454-62. doi: $10.1038 /$ nri1111

4. Marsh DG, Neely JD, Breazeale DR, Ghosh B, Freidhoff LR, Ehrlich-Kautzky E, et al. Linkage analysis of IL4 and other chromosome 5q31.1 markers and total serum immunoglobulin E concentrations. Science. (1994) 264:1152-6. doi: 10.1126/science.81 78175

5. Meyers JH, Chakravarti S, Schlesinger D, Illes Z, Waldner H, Umetsu SE, et al. TIM-4 is the ligand for TIM-1, and the TIM-1-TIM-4 interaction regulates T cell proliferation. Nat Immunol. (2005) 6:455-64. doi: 10.1038/ni1185

6. Abe Y, Kamachi F, Kawamoto T, Makino F, Ito J, Kojima Y, et al. TIM-4 has dual function in the induction and effector phases of murine arthritis. J Immunol. (2013) 191:4562-72. doi: 10.4049/jimmunol.12 03035

7. Mizui M, Shikina T, Arase H, Suzuki K, Yasui T, Rennert PD, et al. Bimodal regulation of $\mathrm{T}$ cell-mediated immune responses by TIM-4. Int Immunol. (2008) 20:695-708. doi: 10.1093/intimm/dxn029

8. Shakhov AN, Rybtsov S, Tumanov AV, Shulenin S, Dean M, Kuprash DV, et al. SMUCKLER/TIM4 is a distinct member of TIM family expressed by stromal cells of secondary lymphoid tissues and associated with lymphotoxin signaling. Eur J Immunol. (2004) 34:494-503. doi: 10.1002/eji.2003 24590

9. Wong K, Valdez PA, Tan C, Yeh S, Hongo JA, Ouyang W. Phosphatidylserine receptor Tim-4 is essential for the maintenance of the homeostatic state of resident peritoneal macrophages. Proc Natl Acad Sci USA. (2010) 107:8712-7. doi: 10.1073/pnas.09109 29107

10. Kobayashi N, Karisola P, Pena-Cruz V, Dorfman DM, Jinushi M, Umetsu SE, et al. TIM-1 and TIM-4 glycoproteins bind phosphatidylserine
Tim-4 in diseases remains unclear, and future research would be expected to provide novel strategies for clinical prediction and treatment.

\section{AUTHOR CONTRIBUTIONS}

WL and LX wrote and revised sections of the manuscript. XLia, $\mathrm{XLiu}$, and YZ collected the related papers, helped to draft, and revise the manuscript. CM participated in the design of the manuscript. LG designed the manuscript and was the major contributor. All authors read and approved the final manuscript.

\section{FUNDING}

This work was supported by the National Natural Science Foundation of China (Nos. 81971480, 81670520, and 81902921), Taishan Scholarship (No. tspd20181201), and Shandong Provincial Key Innovation project (No. 2018YFJH0503). Thanks for the supporting from Collaborative Innovation Center of Technology and Equipment for Biological Diagnosis and Therapy in Universities of Shandong. and mediate uptake of apoptotic cells. Immunity. (2007) 27:927-40. doi: 10.1016/j.immuni.2007.11.011

11. Zhang X, Gu J, Zhou L, Mi QS. TIM-4 is expressed on invariant NKT cells but dispensable for their development and function. Oncotarget. (2016) 7:71099-111. doi: 10.18632/oncotarget.12153

12. Feng BS, Chen X, He SH, Zheng PY, Foster J, Xing Z, et al. Disruption of Tcell immunoglobulin and mucin domain molecule (TIM)-1/TIM4 interaction as a therapeutic strategy in a dendritic cell-induced peanut allergy model. J Allergy Clin Immunol. (2008) 122:55-61;61.e1-7. doi: 10.1016/j.jaci.2008. 04.036

13. Kim HS, Kim HS, Lee CW, Chung DH. T cell Ig domain and mucin domain 1 engagement on invariant NKT cells in the presence of TCR stimulation enhances IL-4 production but inhibits IFN-gamma production. J Immunol. (2010) 184:4095-106. doi: 10.4049/jimmunol.0901991

14. Xu LY, Qi JN, Liu X, Ma HX, Yuan W, Zhao PQ, et al. Tim-4 inhibits NO generation by murine macrophages. PLoS ONE. (2015) 10:e0124771. doi: 10.1371/journal.pone.0124771

15. Xu L, Qi J, Zhao P, Liang X, Ju Y, Liu P, et al. T cell immunoglobulin- and mucin-domain-containing molecule-4 attenuates concanavalin A-induced hepatitis by regulating macrophage. J Leukoc Biol. (2010) 88:329-36. doi: 10.1189/jlb.1209797

16. Baghdadi M, Yoneda A, Yamashina T, Nagao H, Komohara Y, Nagai S, et al. TIM-4 glycoprotein-mediated degradation of dying tumor cells by autophagy leads to reduced antigen presentation and increased immune tolerance. Immunity. (2013) 39:1070-81. doi: 10.1016/j.immuni.2013.09.014

17. Yang B, Luo Y, Liu Z, Yang P, Gui Y. Probiotics SOD inhibited food allergy via downregulation of STAT6-TIM4 signaling on DCs. Mol Immunol. (2018) 103:71-7. doi: 10.1016/j.molimm.2018.09.001

18. Liu ZQ, Li MG, Geng XR, Liu J, Yang G, Qiu SQ, et al. Vitamin D regulates immunoglobulin mucin domain molecule-4 expression in dendritic cells. Clin Exp Allergy. (2017) 47:656-64. doi: 10.1111/cea.12894

19. Zhang Q, Wang H, Wu X, Liu B, Liu W, Wang R, et al. TIM-4 promotes the growth of non-small-cell lung cancer in a RGD motif-dependent manner. $\mathrm{Br}$ J Cancer. (2015) 113:1484-92. doi: 10.1038/bjc.2015.323

20. Tan $\mathrm{X}$, Zhang $\mathrm{Z}$, Yao $\mathrm{H}$, Shen $\mathrm{L}$. Tim-4 promotes the growth of colorectal cancer by activating angiogenesis and recruiting tumor-associated macrophages via the PI3K/AKT/mTOR signaling 
pathway. Cancer Lett. (2018) 436:119-28. doi: 10.1016/j.canlet.2018.0 8.012

21. Dorfman DM, Hornick JL, Shahsafaei A, Freeman GJ. The phosphatidylserine receptors, $\mathrm{T}$ cell immunoglobulin mucin proteins 3 and 4, are markers of histiocytic sarcoma and other histiocytic and dendritic cell neoplasms. Hum Pathol. (2010) 41:1486-94. doi: 10.1016/j.humpath.2010.04.005

22. Fisher DT, Appenheimer MM, Evans SS. The two faces of IL-6 in the tumor microenvironment. Semin Immunol. (2014) 26:38-47. doi: 10.1016/j.smim.2014.01.008

23. Seoane J, Gomis RR. TGF- $\beta$ Family Signaling in Tumor Suppression and Cancer Progression. Cold Spring Harb Perspect Biol. (2017) 9:a022277. doi: 10.1101/cshperspect.a022277

24. Liu W, Bai F, Wang H, Liang Y, Du X, Liu C, et al. Tim-4 inhibits NLRP3 inflammasome via the LKB1/AMPKalpha pathway in macrophages. J Immunol. (2019) 203:990-1000. doi: 10.4049/jimmunol.1900117

25. Yang B, Li LJ, Xu LZ, Liu JQ, Zhang HP, Geng XR, et al. Histone acetyltransferease p300 modulates TIM4 expression in dendritic cells. Sci Rep. (2016) 6:21336. doi: 10.1038/srep21336

26. Jiang R, Jiang Y, Xia P, Luo G, Huang W, Hu Z, et al. Cigarette smoke extract promotes TIM4 expression in murine dendritic cells leading to Th2 polarization through ERK-dependent pathways. Int Arch Allergy Immunol. (2019) 178:219-28. doi: 10.1159/000494505

27. Miyanishi M, Tada K, Koike M, Uchiyama Y, Kitamura T, Nagata S. Identification of Tim4 as a phosphatidylserine receptor. Nature. (2007) 450:435-9. doi: 10.1038/nature06307

28. Yamanishi Y, Kitaura J, Izawa K, Kaitani A, Komeno Y, Nakamura M, et al. TIM1 is an endogenous ligand for LMIR5/CD300b: LMIR5 deficiency ameliorates mouse kidney ischemia/reperfusion injury. J Exp Med. (2010) 207:1501-11. doi: 10.1084/jem.20090581

29. Rodriguez-Manzanet R, Meyers JH, Balasubramanian S, Slavik J, Kassam N, Dardalhon V, et al. TIM-4 expressed on APCs induces T cell expansion and survival. J Immunol. (2008) 180:4706-13. doi: 10.4049/jimmunol.180.7.4706

30. Cao W, Ryan M, Buckley D, O'Connor R, Clarkson MR. Tim-4 inhibition of $\mathrm{T}$-cell activation and $\mathrm{T}$ helper type 17 differentiation requires both the immunoglobulin $\mathrm{V}$ and mucin domains and occurs via the mitogen-activated protein kinase pathway. Immunology. (2011) 133:179-89. doi: 10.1111/j.1365-2567.2011.03424.x

31. Ge RT, Zeng L, Mo LH, Xu LZ, Zhang HP, Yu HQ, et al. Interaction of TIM4 and TIM3 induces T helper 1 cell apoptosis. Immunol Res. (2016) 64:470-5. doi: 10.1007/s12026-015-8702-9

32. Nakahara T, Moroi Y, Uchi H, Furue M. Differential role of MAPK signaling in human dendritic cell maturation and Th1/Th2 engagement. J Dermatol Sci. (2006) 42:1-11. doi: 10.1016/j.jdermsci.2005.11.004

33. Dong C, Davis RJ, Flavell RA. MAP kinases in the immune response. Annu Rev Immunol. (2002) 20:55- 72. doi: 10.1146/annurev.immunol.20.091301.131133

34. Yamashita M, Shinnakasu R, Asou H, Kimura M, Hasegawa A, Hashimoto K, et al. Ras-ERK MAPK cascade regulates GATA3 stability and Th2 differentiation through ubiquitin-proteasome pathway. J Biol Chem. (2005) 280:29409-19. doi: 10.1074/jbc.M5023 33200

35. Xu L, Zhao P, Xu Y, Gao L, Wang H, Jia X, et al. Tim-4 protects mice against lipopolysaccharide-induced endotoxic shock by suppressing the NF-кB signaling pathway. Lab Invest. (2016) 96:1189-97. doi: 10.1038/labinvest.2016.94

36. Tiegs G, Hentschel J, Wendel A. A T cell-dependent experimental liver injury in mice inducible by concanavalin A. J Clin Invest. (1992) 90:196-203. doi: 10.1172/JCI115836

37. Hatano M, Sasaki S, Ohata S, Shiratsuchi Y, Yamazaki T, Nagata K, et al. Effects of Kupffer cell-depletion on concanavalin A-induced hepatitis. Cell Immunol. (2008) 251:25-30. doi: 10.1016/j.cellimm.2008.02.003

38. Segawa K, Nagata S. An apoptotic 'Eat Me' signal: phosphatidylserine exposure. Trends Cell Biol. (2015) 25:639-50. doi: 10.1016/j.tcb.2015.08.003

39. Yanagihashi Y, Segawa K, Maeda R, Nabeshima YI, Nagata S. Mouse macrophages show different requirements for phosphatidylserine receptor Tim4 in efferocytosis. Proc Natl Acad Sci USA. (2017) 114:8800-5. doi: 10.1073/pnas.1705365114

40. Santiago C, Ballesteros A, Martinez-Munoz L, Mellado M, Kaplan GG, Freeman GJ, et al. Structures of T cell immunoglobulin mucin protein 4 show a metal-Ion-dependent ligand binding site where phosphatidylserine binds. Immunity. (2007) 27:941-51. doi: 10.1016/j.immuni.2007.11.008

41. Tietjen GT, Gong Z, Chen CH, Vargas E, Crooks JE, Cao KD, et al. Molecular mechanism for differential recognition of membrane phosphatidylserine by the immune regulatory receptor Tim4. Proc Natl Acad Sci USA. (2014) 111:E1463-72. doi: 10.1073/pnas.1320174111

42. Park D, Hochreiter-Hufford A, Ravichandran KS. The phosphatidylserine receptor TIM-4 does not mediate direct signaling. Curr Biol. (2009) 19:34651. doi: $10.1016 /$ j.cub.2009.01.042

43. Flannagan RS, Canton J, Furuya W, Glogauer M, Grinstein S. The phosphatidylserine receptor TIM4 utilizes integrins as coreceptors to effect phagocytosis. Mol Biol Cell. (2014) 25:1511-22. doi: 10.1091/mbc.e13-04-0212

44. Lee J, Park B, Moon B, Park J, Moon H, Kim K, et al. A scaffold for signaling of Tim-4-mediated efferocytosis is formed by fibronectin. Cell Death Differ. (2019) 26:1646-55. doi: 10.1038/s41418-018-0238-9

45. Dick SA, Macklin JA, Nejat S, Momen A, Clemente-Casares X, Althagafi MG, et al. Self-renewing resident cardiac macrophages limit adverse remodeling following myocardial infarction. Nat Immunol. (2019) 20:29-39. doi: 10.1038/s41590-018-0272-2

46. Shaw TN, Houston SA, Wemyss K, Bridgeman HM, Barbera TA, ZangerleMurray T, et al. Tissue-resident macrophages in the intestine are long lived and defined by Tim-4 and CD4 expression. J Exp Med. (2018) 215:1507-18. doi: 10.1084/jem.20180019

47. Chiaranunt P, Burrows K, Ngai L, Mortha A. Isolation of mononuclear phagocytes from the mouse gut. Methods Enzymol. (2020) 632:67-90. doi: 10.1016/bs.mie.2019.10.004

48. Chen Q, Ruedl C. Obesity retunes turnover kinetics of tissue-resident macrophages in fat. J Leukoc Biol. (2020). doi: 10.1002/JLB.1MA1219-275R. [Epub ahead of print].

49. Yeung MY, McGrath MM, Nakayama M, Shimizu T, Boenisch O, Magee $\mathrm{CN}$, et al. Interruption of dendritic cell-mediated TIM-4 signaling induces regulatory $\mathrm{T}$ cells and promotes skin allograft survival. J Immunol. (2013) 191:4447-55. doi: 10.4049/jimmunol.1300992

50. Li J, Zhao X, Liu X, Liu H. Disruption of TIM-4 in dendritic cell ameliorates hepatic warm IR injury through the induction of regulatory T cells. Mol Immunol. (2015) 66:117-25. doi: 10.1016/j.molimm.2015.02.004

51. Li LJ, Zeng L, Li XX, Mo LH, Geng XR, Zheng PY, et al. Induction of colitis in mice with food allergen-specific immune response. Sci Rep. (2016) 6:32765. doi: 10.1038/srep32765

52. Zhang X, Liu Q, Wang J, Li G, Weiland M, Yu FS, et al. TIM-4 is differentially expressed in the distinct subsets of dendritic cells in skin and skin-draining lymph nodes and controls skin Langerhans cell homeostasis. Oncotarget. (2016) 7:37498-512. doi: 10.18632/oncotarget.9546

53. Thornley TB, Fang Z, Balasubramanian S, Larocca RA, Gong W, Gupta S, et al. Fragile TIM-4-expressing tissue resident macrophages are migratory and immunoregulatory. J Clin Invest. (2014) 124:3443-54. doi: 10.1172/JCI 73527

54. Ding Q, Mohib K, Kuchroo VK, Rothstein DM. TIM-4 identifies IFNgamma-expressing proinflammatory B effector 1 cells that promote tumor and allograft rejection. J Immunol. (2017) 199:2585-95. doi: 10.4049/jimmunol.1602107

55. Nakae S, Iikura M, Suto H, Akiba H, Umetsu DT, Dekruyff RH, et al. TIM-1 and TIM-3 enhancement of Th2 cytokine production by mast cells. Blood. (2007) 110:25658. doi: 10.1182/blood-2006-11-0p 58800

56. Li L, Mo L, Hao H, Yang W, Zhou Q, Xue F, et al. Flagellin modulates TIM4 expression in mast cells. Cell Biol Int. (2014) 38:1330-6. doi: 10.1002/cbin. 10330

57. Chae SC, Song JH, Lee YC, Kim JW, Chung HT. The association of the exon 4 variations of Tim-1 gene with allergic diseases in a Korean population. Biochem Biophys Res Commun. (2003) 312:346-50. doi: 10.1016/j.bbrc.2003.10.125

58. Liu Q, Shang L, Li J, Wang P, Li H, Wei C, et al. A functional polymorphism in the TIM-1 gene is associated with asthma in a Chinese Han population. Int Arch Allergy Immunol. (2007) 144:197-202. doi: 10.1159/0001 03992

59. Cai PC, Hu LH, Cui TP, Li YR, Chen FH, Wu QW. Association of TIM4 promoter polymorphism-1419G $>$ A with childhood asthma 
in a Chinese Han population. Tissue Antigens. (2009) 74:11-6. doi: 10.1111/j.1399-0039.2009.01240.x

60. Xu L, Zhang M, Ma W, Jin S, Song W, He S. Cockroach allergen Bla g 7 promotes TIM4 expression in dendritic cells leading to Th2 polarization. Mediators Inflamm. (2013) 2013:983149. doi: 10.1155/2013/983149

61. Cardona ID, Cho SH, Leung DY. Role of bacterial superantigens in atopic dermatitis: implications for future therapeutic strategies. Am J Clin Dermatol. (2006) 7:273-9. doi: 10.2165/00128071-200607050-00001

62. Aprahamian T, Rifkin I, Bonegio R, Hugel B, Freyssinet JM, Sato K, et al. Impaired clearance of apoptotic cells promotes synergy between atherogenesis and autoimmune disease. J Exp Med. (2004) 199:1121-31. doi: 10.1084 /jem. 20031557

63. Munoz LE, Lauber K, Schiller M, Manfredi AA, Herrmann M. The role of defective clearance of apoptotic cells in systemic autoimmunity. Nat Rev Rheumatol. (2010) 6:280-9. doi: 10.1038/nrrheum.2010.46

64. Fang XY, Xu WD, Pan HF, Leng RX, Ye DQ. Novel insights into Tim4 function in autoimmune diseases. Autoimmunity. (2015) 48:189-95. doi: $10.3109 / 08916934.2014 .983266$

65. Zhao P, Xu L, Wang P, Liang X, Qi J, Liu P, et al. Increased expression of human T-cell immunoglobulin- and mucin-domain-containing molecule4 in peripheral blood mononuclear cells from patients with system lupus erythematosus. Cell Mol Immunol. (2010) 7:152-6. doi: 10.1038/cmi.2009.118

66. Liu B, Liu W, Wang R, Shu Q, Zhang X, Fan X, et al. Promoter polymorphisms of the TIM-4 gene are correlated with disease activity in patients with systemic lupus erythematosus. Int J Immunogenet. (2017) 44:122-8. doi: 10.1111/iji.12316

67. Miyanishi M, Segawa K, Nagata S. Synergistic effect of Tim4 and MFG-E8 null mutations on the development of autoimmunity. Int Immunol. (2012) 24:551-9. doi: 10.1093/intimm/dxs064

68. Rodriguez-Manzanet R, Sanjuan MA, Wu HY, Quintana FJ, Xiao S, Anderson $\mathrm{AC}$, et al. $\mathrm{T}$ and $\mathrm{B}$ cell hyperactivity and autoimmunity associated with niche-specific defects in apoptotic body clearance in TIM-4-deficient mice. Proc Natl Acad Sci USA. (2010) 107:8706-11. doi: 10.1073/pnas.09103 59107

69. Rhodes B, Vyse TJ. General aspects of the genetics of SLE. Autoimmunity. (2007) 40:550-9. doi: 10.1080/08916930701510657

70. Xu J, Yang Y, Liu X, Wang Y. Genetic variation and significant association of polymorphism rs7700944 G>A of TIM-4 gene with rheumatoid arthritis susceptibility in Chinese Han and Hui populations. Int J Immunogenet. (2012) 39:409-13. doi: 10.1111/j.1744-313X.2012.01103.x

71. Zakeri Z, Hashemi M, Pourhosseini SM, Eskandari-Nasab E, Bahari G, Taheri M. Association between the rs7700944 polymorphism in the TIM-4 gene and rheumatoid arthritis in Zahedan, southeast Iran. Rev Bras Reumatol. (2013) 53:341-5. doi: 10.1016/S2255-5021(13)70 046-4

72. Mosaad YM, El-Bassiony SR, El-Ghaweet AE, Elhindawy MM, El-Deek BS, Sultan WA. TIM-1 rs41297579 G>A (-1454) and TIM-4 rs7700944 gene polymorphisms as possible risk factor for rheumatoid arthritis: relation to activity and severity. Int J Immunogenet. (2015) 42:254-64. doi: $10.1111 /$ iji. 12201

73. Xu L, Xiao H, Xu M, Zhou C, Yi L, Liang H. Glioma-derived T cell immunoglobulin- and mucin domain-containing molecule-4 (TIM4) contributes to tumor tolerance. J Biol Chem. (2011) 286:36694-9. doi: $10.1074 /$ jbc.M111.292540

74. Albacker LA, Karisola P, Chang YJ, Umetsu SE, Zhou M, Akbari $\mathrm{O}$, et al. TIM-4, a receptor for phosphatidylserine, controls adaptive immunity by regulating the removal of antigen-specific T cells. J Immunol. (2010) 185:6839-49. doi: 10.4049/jimmunol.10 01360

75. Etzerodt A, Moulin M, Doktor TK, Delfini M, Mossadegh-Keller N, Bajenoff $\mathrm{M}$, et al. Tissue-resident macrophages in omentum promote metastatic spread of ovarian cancer. J Exp Med. (2020) 217:e20191869. doi: 10.1084/jem.20191869

76. Cheng L, Ruan Z. Tim-3 and Tim- 4 as the potential targets for antitumor therapy. Hum Vaccin Immunother. (2015) 11:2458-62. doi: 10.1080/21645515.2015.1056953

77. Jinushi M, Sato M, Kanamoto A, Itoh A, Nagai S, Koyasu S, et al. Milk fat globule epidermal growth factor- 8 blockade triggers tumor destruction through coordinated cell-autonomous and immune-mediated mechanisms. $J$ Exp Med. (2009) 206:1317-26. doi: 10.1084/jem.20082614

78. Baghdadi M, Nagao H, Yoshiyama H, Akiba H, Yagita H, Dosaka-Akita H, et al. Combined blockade of TIM-3 and TIM-4 augments cancer vaccine efficacy against established melanomas. Cancer Immunol Immunother. (2013) 62:629-37. doi: 10.1007/s00262-012-1371-9

79. Sun HW, Wu C, Tan HY, Wang QS. A new development of FGCC' siRNA blocking interaction of Tim-1 and Tim-4 can enhance DC vaccine against gastric cancer. Hepatogastroenterology. (2012) 59:2677-82. doi: 10.5754/hge11620

80. Liu W, Wang H, Bai F, Ding L, Huang Y, Lu C, et al. IL-6 promotes metastasis of non-small-cell lung cancer by up-regulating TIM-4 via NF-кB. Cell Prolif. (2020). doi: 10.1111/cpr.12776. [Epub ahead of print].

81. Li W, Li X, Xu S, Ma X, Zhang Q. Expression of Tim4 in Glioma and its regulatory role in LN-18 glioma cells. Med Sci Monit. (2016) 22:77-82. doi: 10.12659/MSM.894963

82. DuPage M, Mazumdar C, Schmidt LM, Cheung AF, Jacks T. Expression of tumour-specific antigens underlies cancer immunoediting. Nature. (2012) 482:405-9. doi: 10.1038/nature10803

83. DuPage M, Cheung AF, Mazumdar C, Winslow MM, Bronson R, Schmidt $\mathrm{LM}$, et al. Endogenous $\mathrm{T}$ cell responses to antigens expressed in lung adenocarcinomas delay malignant tumor progression. Cancer Cell. (2011) 19:72-85. doi: 10.1016/j.ccr.2010.11.011

84. Rhein BA, Brouillette RB, Schaack GA, Chiorini JA, Maury W. Characterization of human and murine T-Cell immunoglobulin mucin domain 4 (TIM-4) IgV domain residues critical for ebola virus entry. J Virol. (2016) 90:6097-111. doi: 10.1128/JVI.00100-16

85. Dragovich MA, Fortoul N, Jagota A, Zhang W, Schutt K, Xu Y, et al. Biomechanical characterization of TIM protein-mediated Ebola virushost cell adhesion. Sci Rep. (2019) 9:267. doi: 10.1038/s41598-018-36 s449-2

86. Sims B, Farrow AL, Williams SD, Bansal A, Krendelchtchikov A, Gu L, et al. Role of TIM-4 in exosome-dependent entry of HIV-1 into human immune cells. Int J Nanomed. (2017) 12:4823-33. doi: 10.2147/IJN.S132762

87. Jemielity S, Wang JJ, Chan YK, Ahmed AA, Li W, Monahan S, et al. TIMfamily proteins promote infection of multiple enveloped viruses through virion-associated phosphatidylserine. PLoS Pathog. (2013) 9:e1003232. doi: 10.1371/journal.ppat.1003232

88. Czuczman MA, Fattouh R, van Rijn JM, Canadien V, Osborne S, Muise AM, et al. Listeria monocytogenes exploits efferocytosis to promote cell-to-cell spread. Nature. (2014) 509:230-4. doi: 10.1038/nature13168

89. Kathiresan S, Willer CJ, Peloso GM, Demissie S, Musunuru K, Schadt EE, et al. Common variants at 30 loci contribute to polygenic dyslipidemia. Nat Genet. (2009) 41:56-65. doi: 10.1038/ng.291

90. Park MH, Kim N, Lee JY, Park HY. Genetic loci associated with lipid concentrations and cardiovascular risk factors in the Korean population. J Med Genet. (2011) 48:10-5. doi: 10.1136/jmg.2010.0 81000

91. Zhang Z, Tao L, Chen Z, Zhou D, Kan M, Zhang D, et al. Association of genetic loci with blood lipids in the Chinese population. PLoS ONE. (2011) 6:e27305. doi: 10.1371/journal.pone.0027305

92. Willer CJ, Schmidt EM, Sengupta S, Peloso GM, Gustafsson S, Kanoni S, et al. Discovery and refinement of loci associated with lipid levels. Nat Genet. (2013) 45:1274-83. doi: 10.1038/ng.2797

93. Li N, van der Sijde MR, LifeLines Cohort Study Group, Bakker SJ, Dullaart RP, van der Harst P, et al. Pleiotropic effects of lipid genes on plasma glucose, HbA1c, and HOMA-IR levels. Diabetes. (2014) 63:3149-58. doi: $10.2337 / \mathrm{db} 13-1800$

94. Zhao $\mathrm{P}$, Wang $\mathrm{H}, \mathrm{Li} \mathrm{T}$, Lei $\mathrm{C}, \mathrm{Xu} \mathrm{X}$, Wang $\mathrm{W}$, et al. Increased $\mathrm{T}$ cell immunoglobulin and mucin domain containing 4 (TIM- 4 ) is negatively correlated with serum concentrations of interleukin- $1 \beta$ in type 2 diabetes. J Diabetes. (2016) 8:199-205. doi: 10.1111/1753-0407. 12276

95. Foks AC, Engelbertsen D, Kuperwaser F, Alberts-Grill N, Gonen A, Witztum JL, et al. Blockade of Tim-1 and Tim- 4 enhances atherosclerosis in low-density lipoprotein receptor-deficient mice. Arterioscler Thromb Vasc Biol. (2016) 36:456-65. doi: 10.1161/ATVBAHA.115.3 06860 
96. Akiyama K, Liang YQ, Isono M, Kato N. Investigation of functional genes at homologous loci identified based on genome-wide association studies of blood lipids via high-fat diet intervention in rats using an in vivo approach. $J$ Atheroscler Thromb. (2015) 22:455-80. doi: 10.5551/jat.27706

97. Schweigert O, Dewitz C, Moller-Hackbarth K, Trad A, Garbers C, RoseJohn S, et al. Soluble $\mathrm{T}$ cell immunoglobulin and mucin domain (TIM)-1 and -4 generated by A Disintegrin and metalloprotease (ADAM)-10 and-17 bind to phosphatidylserine. Biochim Biophys Acta. (2014) 1843:275-87. doi: 10.1016/j.bbamcr.2013.11.014

98. Xu L, Ye Z, Wang B, Huang Y, Zhou L, Liu C, et al. Tim-4 expression increases in ischemic stroke patients and is associated with poor outcome. $J$ Neuroimmunol. (2018) 316:1-6. doi: 10.1016/j.jneuroim.2017.11.017

99. Chen D, He J, Lu C, Zhou J, Fang K, Liu X, et al. Increased expression of T cell immunoglobulin and mucin domain 4 is positively associated with the disease severity of patients with ankylosing spondylitis. Inflammation. (2015) 38:935-40. doi: 10.1007/s10753-014-0055-3

Conflict of Interest: The authors declare that the research was conducted in the absence of any commercial or financial relationships that could be construed as a potential conflict of interest.

Copyright (c) $2020 \mathrm{Liu}, \mathrm{Xu}$, Liang, Liu, Zhao, Ma and Gao. This is an open-access article distributed under the terms of the Creative Commons Attribution License (CC $B Y)$. The use, distribution or reproduction in other forums is permitted, provided the original author(s) and the copyright owner(s) are credited and that the original publication in this journal is cited, in accordance with accepted academic practice. No use, distribution or reproduction is permitted which does not comply with these terms. 